C-A/AP/\#119

October 2003

FY03 Polarized Proton Ramp Optics Analysis

J. van Zeijts

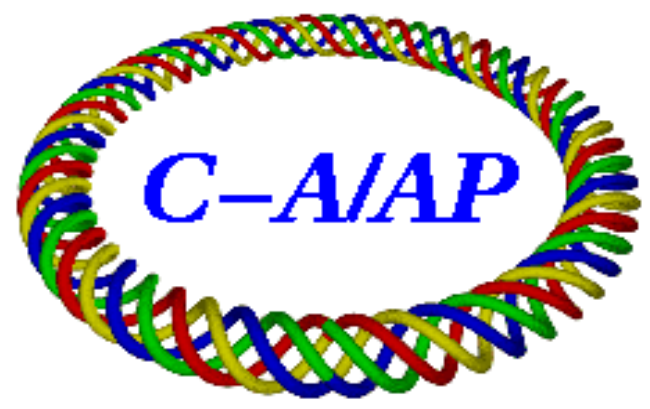

Collider-Accelerator Department Brookhaven National Laboratory

Upton, NY 11973 


\title{
FY03 Polarized Proton Ramp Optics Analysis
}

\author{
J. van Zeijts, BNL, Upton, USA*
}

\section{Abstract}

For the recent RHIC polarized-proton (pp) ramps the beam was first accelerated with constant $\beta^{*}$ and subsequently squeezed at flattop energy. This allowed a separate analysis of optics errors during the acceleration part and during the squeeze part of the ramp. The main observed effects were a constant tune offset during acceleration, and the neccessity to add a tune swing of $\approx 0.1$ units (Figures 4,5$)$ along the squeeze, to keep the measured tunes at the desired values $(28.23,29.22)$. Based on measured tunes and measured phase-advance[1] at BPMs, changes to quadrupole and dipole transfer-functions are suggested, which greatly reduce this disparity. We show the associated changes in lattice functions and discuss implications for the upcoming FY04 ramps. This analysis only takes linear uncoupled effects into account.

\section{INTRODUCTION}

\subsection{Ramp Design}

The pp ramp consisted of constant $\beta^{*}$ acceleration to flattop $B \rho$ for the first 140 seconds, and, starting at 150 seconds, a $\beta^{*}$ squeeze at fixed $B \rho$ to the final $\beta^{*}$ depending on IP, see Figure 1.

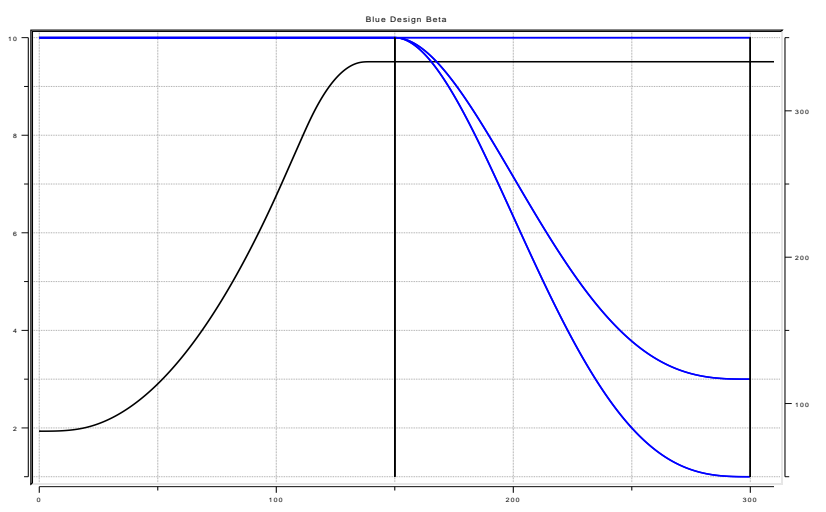

Figure 1: pp Ramp Design: $\beta^{*}(t)$ (blue) and $B \rho(t)$ (black)

\subsection{Online Model}

For FY03, the RHIC online model and RampManager[2] where set up with individual quadrupole transfer-functions and extra focussing terms due to main dipole $b_{2}(I)$ (sextupole) component feed-down. Figure 2 shows the predicted tunes without and with the dipole feed-down where we used a constant offset along the ramp of $\Delta x=$

* Work performed under the auspices of the US Department of Energy
$2.15 \mathrm{~mm}$. A significant improvement is seen in the modeled tune seperation. The $b_{2}$ term is incorperated in the 'design' model where tunes are adjusted to design values using the main-quad bus knobs. Each magnet receives its 'design' setting from this model. During commissioning, tunes are adjusted by introducting 'trim' settings for magnets located only on the main-quad buses. The resulting optics is modeled in the 'trim' model, whose deviaton from design is, in some sense, a measure of our understanding about the machine optics. For the pp ramp the main feature is the negative tune swing during the squeeze.

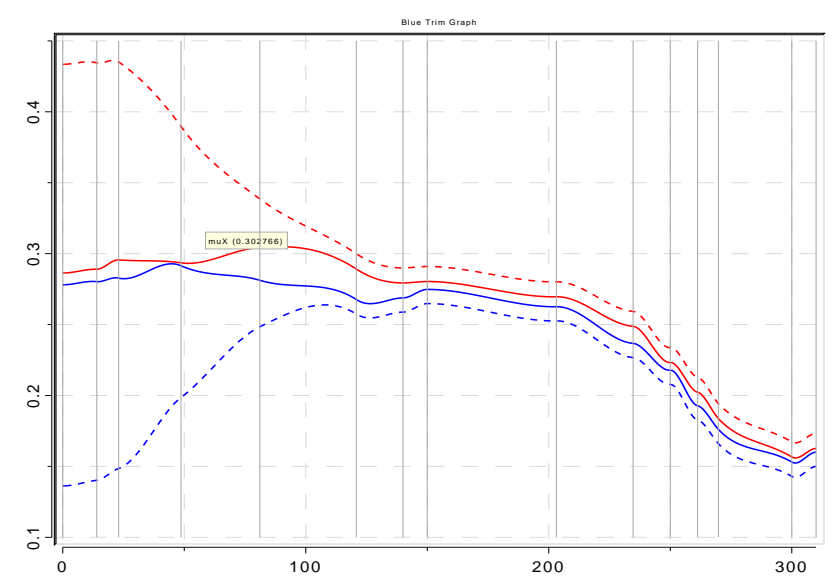

Figure 2: Blue tunes: No $b_{2}$ feed-down (dashed), with $b_{2}$ feed-down correction (solid) assuming $\Delta x=2.15 \mathrm{~mm}$

\section{MODIFIED TRANSFER-FUNCTIONS}

In this section we model errors in transfer-functions (T) for the main dipoles and the main quadrupoles. From the equation for the integrated magnetic field strength:

$$
\alpha \times B \rho=\int B d l=I_{d} \times T_{d}\left(I_{d}\right)
$$

where $\alpha$ is the dipole angle, it follows that the effect of a relative change in the dipole transfer-function on $B \rho$ is:

$$
\frac{\Delta B \rho}{B \rho}=-\frac{\Delta \alpha}{\alpha}+\frac{\Delta I_{d}}{I_{d}}+\frac{\Delta T_{d}}{T_{d}}
$$

Similarly, the effects of a relative change in the quadrupole transfer-functions on quadrupole strength is:

$$
\frac{\Delta \mathrm{K}}{\mathrm{K}}=-\frac{\Delta B \rho}{B \rho}+\frac{\Delta I_{q}}{I_{q}}+\frac{\Delta T_{q}}{T_{q}}
$$

We group the changes in three sets: $\Delta B \rho / B \rho$, resulting from an assumed error in the dipole transfer-function; A second parameter applied to all quadrupoles only powered 
by the main-quad busses $\Delta \mathrm{K}_{\mathrm{arc}} / \mathrm{K}_{\mathrm{arc}}$, resulting from assumed errors in arc quadrupoles transfer-functions. These two sets include magnets which have not all been measured cold. The final parameter $\Delta \mathrm{K}_{\mathrm{ir}} / \mathrm{K}_{\mathrm{ir}}$ is applied to all quadrupoles in the IRs, in principle we could choose a unique number for each IR magnet, but this is prohibited by the limited data available. Since we have found no reasonable explanation for the tune discrepancies using only errors in power supply current, we are assuming no power supply errors: $\Delta I=0$, and no dipole angle errors: $\Delta \alpha=0$. Empirically we arrived at the solution:

Table 1: $\Delta \mathrm{K} / \mathrm{K}$

\begin{tabular}{|l|c|c|}
\hline What & Blue & Yellow \\
\hline$\Delta \mathrm{K}_{\text {arc }} / \mathrm{K}_{\text {arc }}$ & -0.00535 & -0.0064 \\
$\Delta \mathrm{K}_{\mathrm{ir}} / \mathrm{K}_{\text {ir }}$ & 0.00285 & 0.0028 \\
\hline
\end{tabular}

From eq. 3 it follows that once we have found solutions for $\Delta \mathrm{K} / \mathrm{K}$, we can use $\Delta B \rho / B \rho$ as a free parameter to choose $\Delta \mathrm{T} / \mathrm{T}$. The 1-parameter solution for the Blue parameters is shown in Figure 3. In particular we can choose

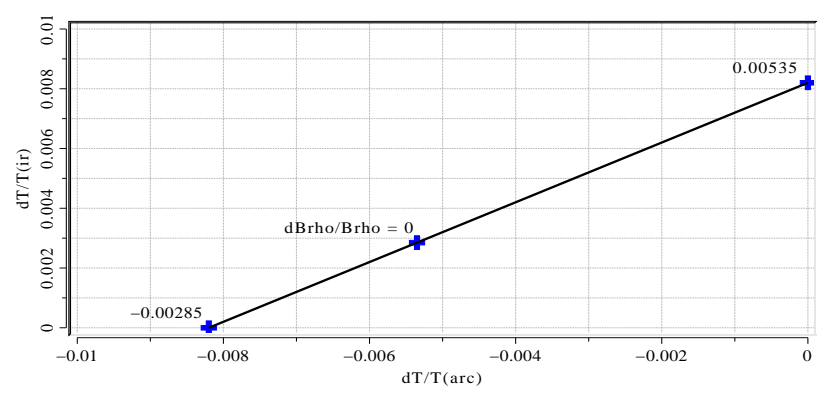

Figure 3: $\Delta T / T($ ir $)$ vs. $\Delta T / T(\operatorname{arc})$.

$\Delta B \rho / B \rho=0$ and set $\Delta T / T=\Delta K / K$. Using these numbers we recalculate the optics along the ramp, and at store. We present the tunes in fig. 4 , and 5. We see that, using this simple assumption, excellent agreement is reached with the measured tunes. A determination of the transfer-functions errors is not possible without extra data fixing the $B \rho$ error.

\subsection{PHASE ADVANCE}

At store we compare measured phase-advance data to the modified model (Figures 6,7,8,9). The comparison is compromised by some suspect data points, but for the most part the agreement of measured wrt. the modified model shows significant better agreement vs. the design model.

\subsection{BETA FUNCTIONS}

The lattice-functions at store of the modified model are compared with the design model. We show Design, Corrected, and the associated $\Delta \beta / \beta$. In addition Table 2 , and 3 give the $\beta^{*}$ at each IP for the modified model.

Assuming the same emittance in both planes and both rings, we compare the fourth root of the product of the Blue

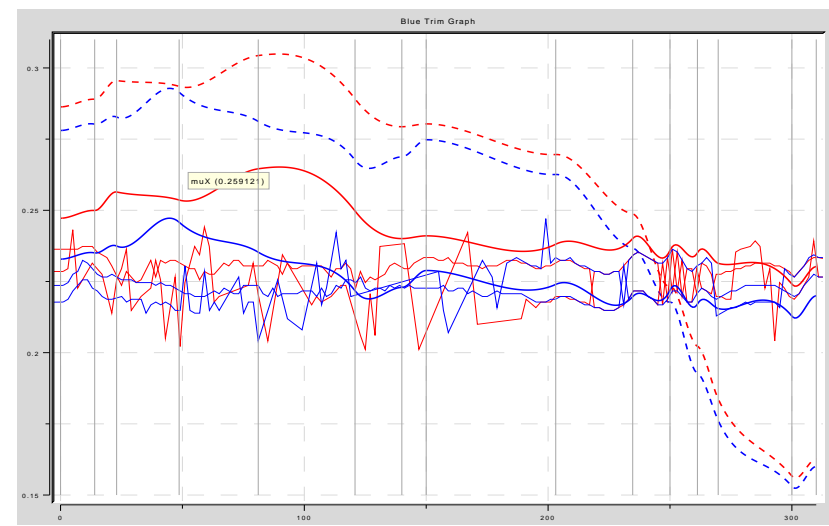

Figure 4: Blue tunes: Model (dashed), with TF dorrection (solid), and Measured

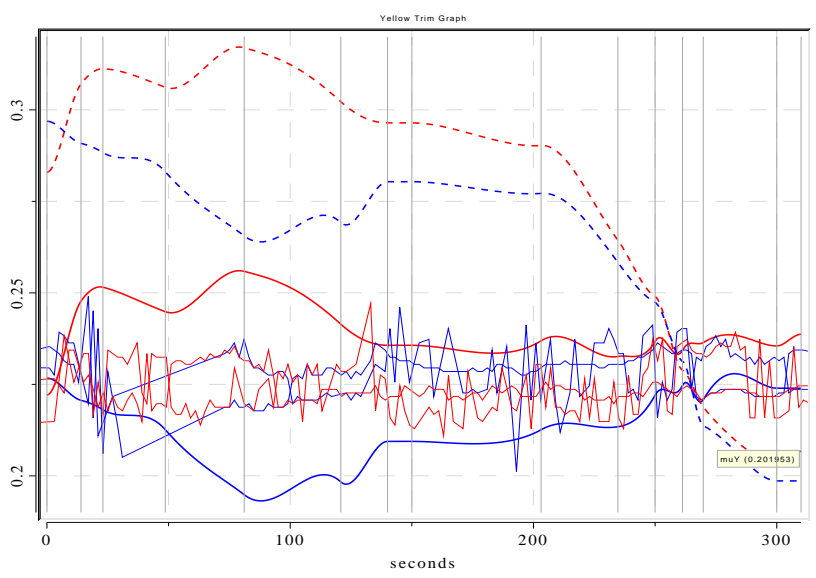

Figure 5: Yellow tunes: Model (dashed), with TF correction (solid), and Measured

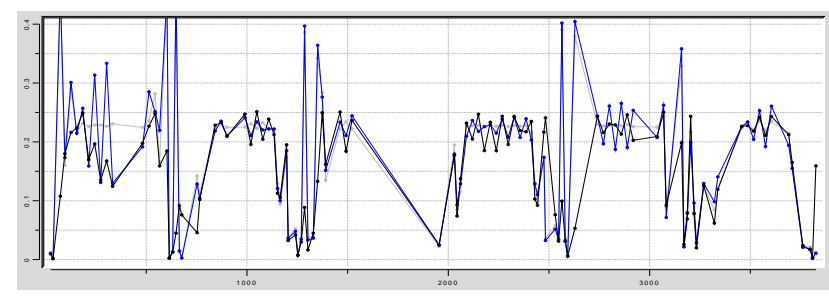

Figure 6: Blue horizontal phase advance between BPMs: Design (grey), corrected TF (blue), and Measured (black).

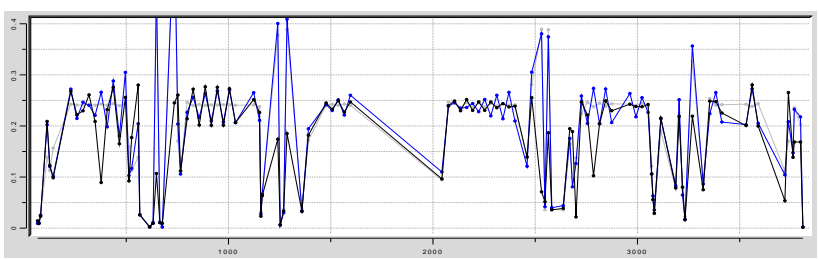

Figure 7: Blue vertical phase advance between BPMs: Design (grey), corrected TF (blue), and Measured (black). 


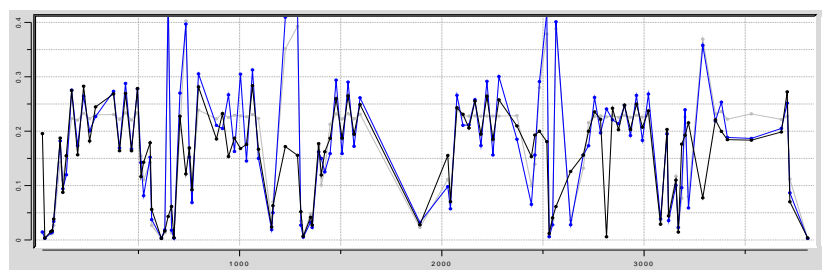

Figure 8: Yellow horizontal phase advance between BPMs: Design (grey), Corrected (blue), and Measured (black).

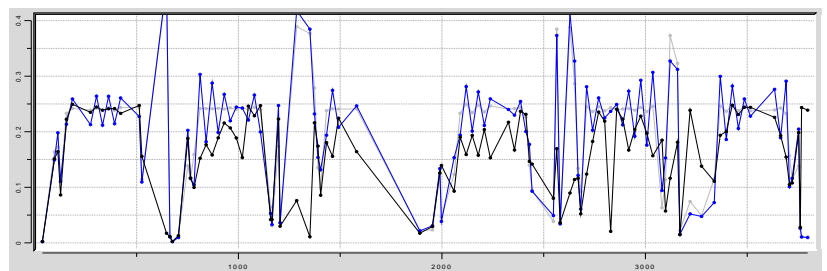

Figure 9: Yellow vertical phase advance between BPMs: Design (grey), Corrected (blue), and Measured (black).

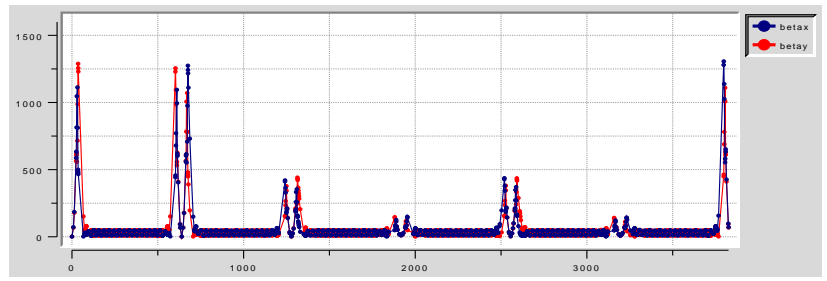

Figure 10: Blue $\beta$ functions (m) for Design model vs. $\mathrm{s}(\mathrm{m})$

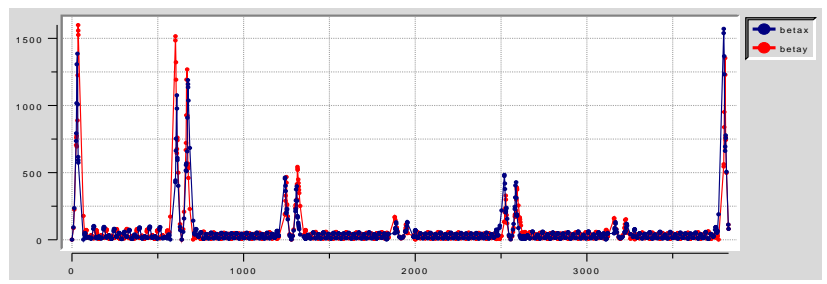

Figure 11: Blue $\beta$ functions with TF corrections

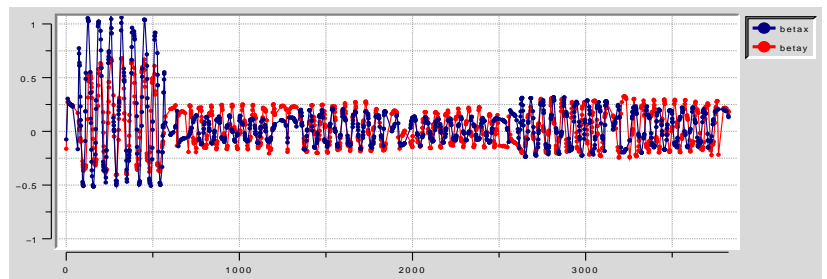

Figure 12: Blue $\Delta \beta / \beta$

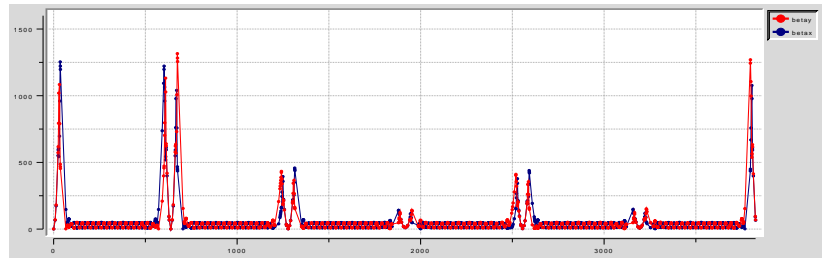

Figure 13: Yellow $\beta$ functions Design model

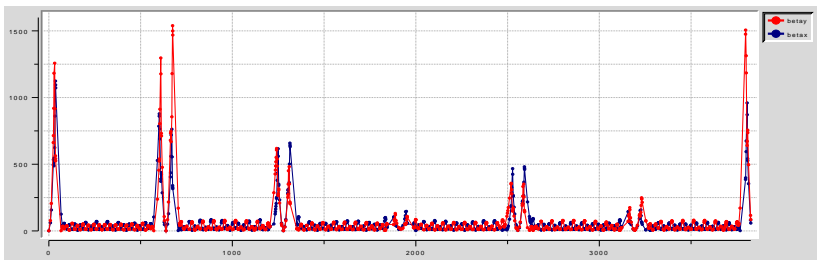

Figure 14: Yellow $\beta$ functions with TF corrections

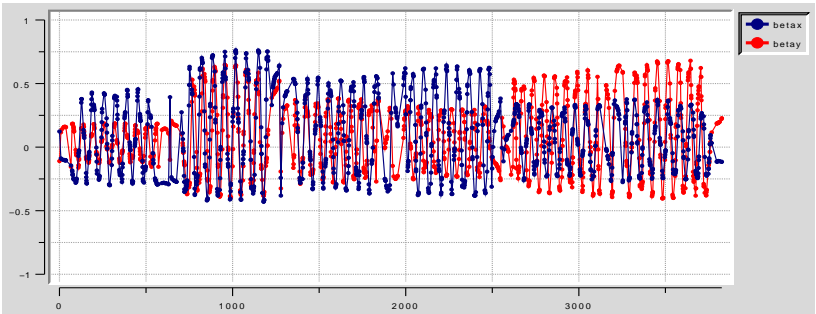

Figure 15: Yellow $\Delta \beta / \beta$

and Yellow $\beta_{x}^{*} \beta_{y}^{*}$ which should, to first order, be the luminosity ratios. The measured ratio between IP $8 / 10=2.3$ [3], close to the corrected model value of 2.25 .

\subsection{RESIDUAL DISPERSION}

Using the same modified optics presented in the previous section, we note a residual dispersion effect. Figures 1619 shows the model, measured and residual dispersion in Blue and Yellow. We can explain this residual dispersion by modifying the angles in the IP region dipoles, a final determination of this effect is lacking.

\section{FY04 AU/AU RAMP}

Applying the same TF correction to the Yellow d/Au run data, gives us some idea of the expected model tunes for the FY04 run. The tunes are shown in Figure 20, and show better, but not perfect, agreement with measured tunes. The $\mathrm{d} / \mathrm{Au}$ store condition were set up with a slight error in the path-length, causing some distortion in the model data.

\section{SUMMARY}

We have suggested corrections in magnet transferfunctions which greatly improve the agreement between the model and measured machine. The corrections involve a relative change in dipole, quad-bus, and IR quadrupole

Table 2: Blue $\beta^{*}$

\begin{tabular}{|l|c|c|c|c|}
\hline IP & Design $\beta$ & $\beta_{x}(m)$ & $\beta_{y}(m)$ & $\beta_{y} \beta_{y}\left(m^{2}\right)$ \\
\hline 6 & 1 & 0.913676 & 0.859847 & 0.786 \\
8 & 1 & 1.23849 & 0.889532 & 1.102 \\
10 & 3 & 2.79955 & 2.45149 & 6.863 \\
2 & 3 & 2.69404 & 3.47097 & 9.351 \\
\hline
\end{tabular}


Table 3: Yellow $\beta^{*}$

\begin{tabular}{|l|c|c|c|c|}
\hline IP & Design $\beta$ & $\beta_{x}(m)$ & $\beta_{y}(m)$ & $\beta_{y} \beta_{y}\left(m^{2}\right)$ \\
\hline 6 & 1 & 1.18728 & 0.90386 & 1.073 \\
8 & 1 & 1.47251 & 0.905545 & 1.333 \\
10 & 3 & 2.21224 & 2.44306 & 5.405 \\
2 & 3 & 3.02624 & 3.8901 & 11.784 \\
\hline
\end{tabular}

Table 4: Luminosity Ratios

\begin{tabular}{|l|c|c|}
\hline IP & $\sqrt[4]{\beta_{x}^{B} \beta_{y}^{B} \beta_{x}^{Y} \beta_{y}^{Y}}(m)$ & wrt. IP 10 \\
\hline 6 & 0.958 & 2.58 \\
8 & 1.10 & 2.25 \\
10 & 2.47 & 1 \\
2 & 3.24 & 1.31 \\
\hline
\end{tabular}

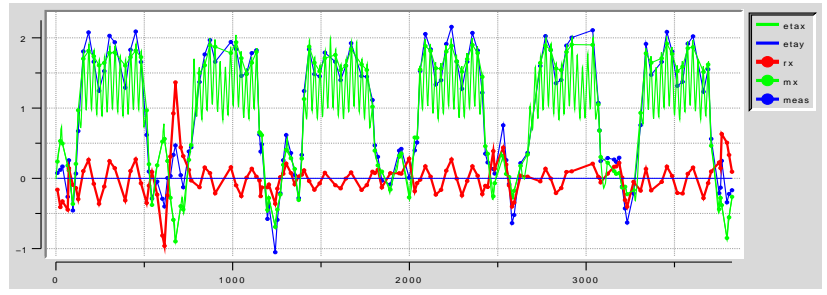

Figure 16: Blue Horizontal Dispersion: Model (Green), Measured (Blue), and Residual (Red)

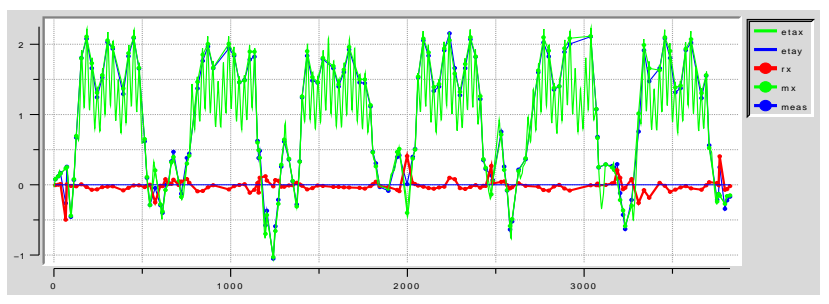

Figure 17: Blue Horizontal Dispersion with DHX angles correction: Model (Green), Measured (Blue), and Residual (Red)

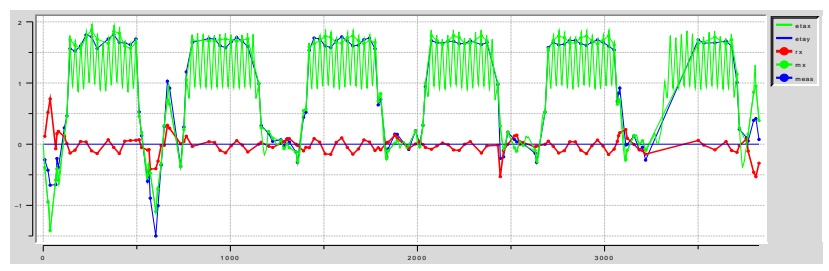

Figure 18: Yellow Horizontal Dispersion: Model (Green), Measured (Blue), and Residual (Red)

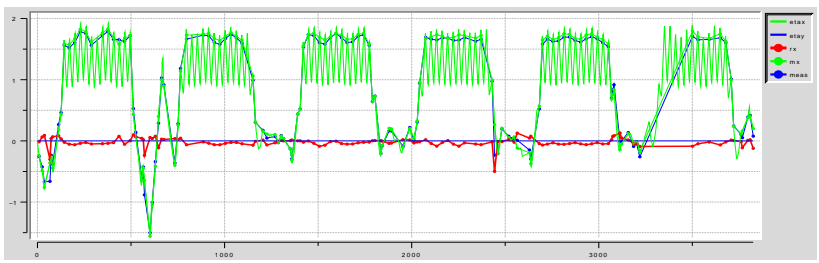

Figure 19: Yellow Horizontal Dispersion with DHX angles correction: Model (Green), Measured (Blue), and Residual (Red)

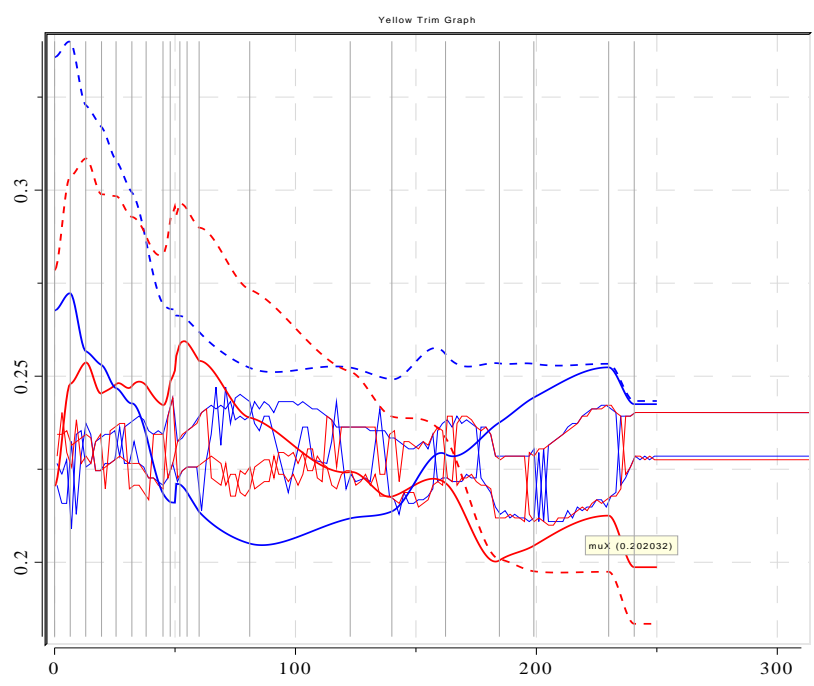

Figure 20: Yellow dAu4 Tunes: Model (Dashed), with TF Correction (Solid), and Measured

transfer-functions and are based on pp ramp measurements at currents of $\approx 2000$ Amps and below. The corrections need to be verified, and most likely updated, for currents up to the Au store conditions. The verification has to involve fixing the $B \rho$ error and probably ramping with constant $\beta^{*}=5 \mathrm{~m}$ after transition, in order to seperate high $\beta$ effects.

A good understanding of the linear optics provides a foundation for any further squeezing below $\beta^{*}=1 \mathrm{~m}$.

\section{ACKNOWLEDGEMENTS}

The author would like to thank Todd Satogate for proof reading and constructive comments. Any missing, or illegible graph markups are the sole responsibility of the author.

\section{REFERENCES}

[1] M. Bai, Measuring Beta Function and Phase Advance in RHIC with an AC Dipole, Proceedings PAC 2003.

[2] J. van Zeijts, Model Driven Ramp Control at RHIC, Proceedings ICALEPCS 2001.

[3] A. Drees, rhic_pp_fy03 elog entry on Sun May 42003. 